Research Journal of Medical Sciences 5 (5): 273-275, 2011

ISSN: $1815-9346$

(C) Medwell Journals, 2011

\title{
Single Implant Retained Mandibular Overdenture with Immediate Loading (Case Report)
}

\author{
${ }^{1}$ Fariborz Vafaee, ${ }^{2}$ Masoumeh Khoshhal, ${ }^{3}$ Sara Tavakolizadeh, \\ ${ }^{4}$ Zahra Ebrahimzadeh, ${ }^{5}$ Mahdi Kadkhodazadeh and ${ }^{2}$ Parviz Torkzaban \\ ${ }^{1}$ Department of Prosthodontics, Dental Research Center, \\ ${ }^{2}$ Department of Periodontics, Dental School, \\ ${ }^{3}$ Department of Prosthodontics, ${ }^{4}$ Department of Perioodontics, \\ Hamadan University of Medical Sciences, Hamadan, Iran \\ ${ }^{5}$ Department of Periodontics, Dental School, \\ Shahid Beheshti University of Medical Sciences, Tehran, Iran
}

\begin{abstract}
Implant therapy can provide a highly successful restoration of both function and esthetics in both completely and partially edentulous patient. To shorten the treatment period and to avoid the edentulous state of patients, it is recommended to perform the surgical techniques that would allow the immediate or early loading of implants. Many patients could not receive implants treatment because of financial problems so we present a case of single implant retained mandibular over denture with 6 months follow up.
\end{abstract}

Key words: Implant, overdenture, stability, bone loss, immediate loading, probing depth

\section{INTRODUCTION}

Many clinicians today recommend implant therapy for patients requiring tooth replacement. This therapy can provide a highly successful restoration of both function and esthetics in both completely and partially edentulous patient (Lee and Agar, 2006; Lee et al., 2009; Cochran, 2006; Ercoli et al., 2006).

In the last 30 years, use of unloaded and submerge implant for achieving osseointegration thought necessary (Stephan et al., 2007). Because of the prolonged period between implant placement and implant loading, problems such as patient and practitioner concerns with the long edentulous state in patients have been continuously debated. To shorten the treatment period and to avoid the edentulous state of patients, numerous studies have been performed on the surgical techniques that would allow the immediate or early loading of implants. Recent studies have shown that there is no difference between the use of 2-stage surgery according to Branemark protocol and one stage treatment protocol with immediate loading that was first arose when Ledermann introduced his modality with edentulous mandible (Lee et al., 2009; Attard and Zarb, 2005; Winter et al., 2004). When there is no indication for fixed restoration because of anatomical, functional and financial problems in mandible, implant-retained overdenture will be an optimal treatment (Stephan et al., 2007; Cannizzaro et al., 2008).
Highly implant success rate have also achieved by lots of studies using 2 or more implant to anchor an overdenture (Stephan et al., 2007; Bergendal and Engquist, 1998; Chiapasco et al., 2001; Payne et al., 2001). Because mandible is hinge-like and its buttressing lingual bone is shock absorbing, use of 2 implant is optimal for support and retention of overdenture and also some researches know this as a standard for edentulous mandible (Lee and Agar, 2006; Sadowsky and Caputo, 2004).

Use of ball 0 -ring attachment could be advantages for implant supported overdentures with regard to optimizing stress and minimizing denture movement in comparison to bar attachments and magnets (Tokuhisa et al., 2003).

Yet many patients could not receive implants treatment because of financial problems and with regard to a recent studies that report immediate loaded single implant retained overdenture as a safe, reliable and cost effective treatment (Chiapasco et al., 2001; Liddelow and Henry, 2007, 2010; Kronstrom and Davis, 2010).

\section{CLINICAL REPORT}

A 45 years old woman presented, complaining of poor retention of mandibular denture and difficulty in eating and speaking. Teeth lost gradually due to caries.

Corresponding Author: Parviz Torkzaban, Department of Periodontics, Dental School, Hamadan University of Medical Sciences, Hamadan, Iran 
The patient had no significant problem in her medical history. About 1 year ago dentures were made for her according to prosthetic condition (neutral zone, occlusal plan and esthetics), dentures are acceptable.

\section{PROCEDURE}

A transparent stent was duplicated from mandibular denture with transparent self-cured acrylic and three holes was drilled in $\mathrm{A}, \mathrm{B}$ and $\mathrm{C}$ positions (according to Misch category) (Misch and Bidez, 2008). These holes was filled by gutta percha (Aria dent, Iran ) and surface was painted by barium sulphate as radio opaque material. Bone condition and essential changes in implant position was determined by CBCT.

There was enough bone for insertion of implant (implantium/simple line II, Dentium; South Korea) with $10 \mathrm{~mm}$ length and $4 \mathrm{~mm}$ width in the $\mathrm{C}$ position.

The stent was used as surgical guide during placement and implant was insered with $30 \mathrm{~N}$ torque (NSK, Surgical Xt. Japan). Primary implant stability was measured with Ossstell (Integration Diagnostic, Gamlestadsvagen, Gotenburg and Sweden) at the end of surgery.

Digital parallel periapical radiography with a positioning stent was taken from patient immediately after the surgery and patient got her denture with proper ball attachment (implantium/simple line 2, Dentium, South Korea) in the first $48 \mathrm{~h}$ after the surgery (Stephan et al., 2007; Chiapasco and Gatti, 2003; Chiapasco et al., 2001) Amoxicilin (to prevent infection) $500 \mathrm{mg} 3$ times a day and Gelofen (to pain control) $400 \mathrm{mg} 4$ times a day was prescribed for patient. She was advised to use chlorhexidine mouth rinse $(0 / 2 \%$, Najo, Iran $) 2$ times a day. Also patient instructed to use soft diet for 6 weeks.

Patient followed for 1, 3 and 6 months after surgery and this parameter was evaluated:

- Marginal bone loss with digital periapical radiography with same positioning stent

- Soft tissue parameters such as probing depth, bleeding on probing with periodontal probe were checked in 4 different sites of the implant $(M, D, B, L)$ 3 and 6 months after surgery

- Implant stability evaluation

\section{RESULTS}

Marginal bone loss was comparable to delay loading of implant and was $0.5 \mathrm{~mm}$ at 6 month, that was acceptable (Misch and Bidez, 2008). There were no signs of BOP and probe depth were not abnormal $(3 \mathrm{~mm})$. ISQ values were $70,72,75,77$ at the surgery, $1,3,6$ months follow up, respectively (Fig. 1-3).

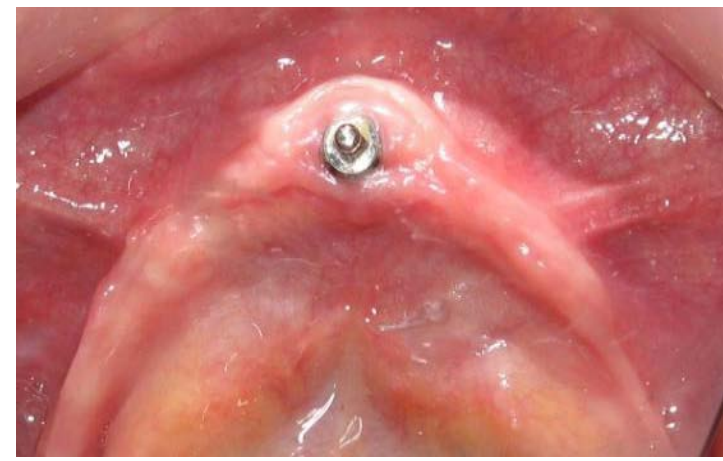

Fig. 1: The 6 months after surgery

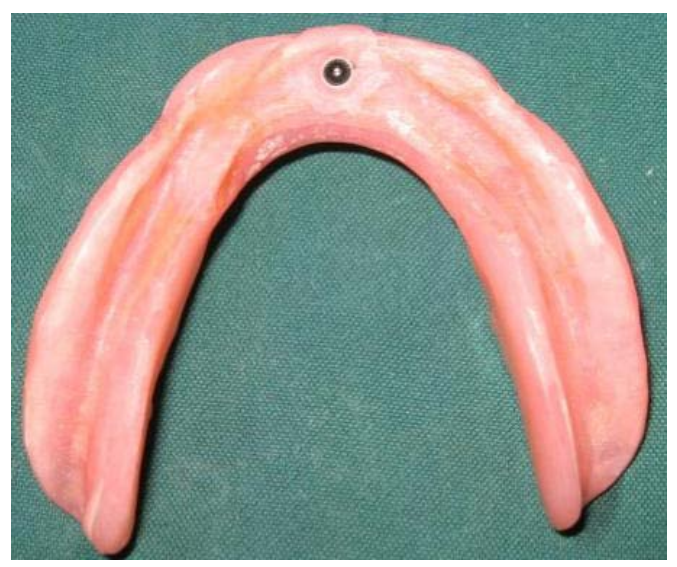

Fig. 2: Mandibular denture

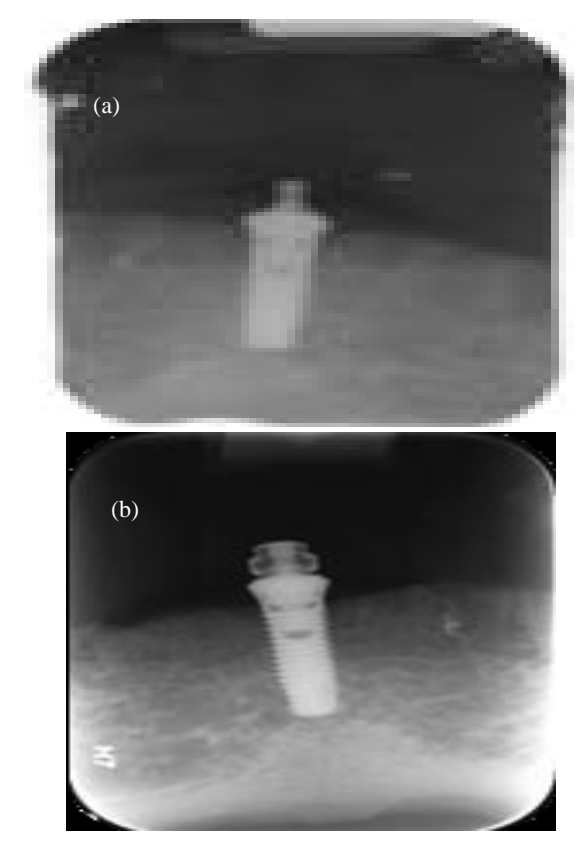

Fig. 3: Peri apical radiograph: immediately (a) after surgery (b) after 6 month 


\section{CONCLUSION}

Regarding to this case, application of one implant because of economic problems of patient is useful and is a starting point for ideal implant supported overdenture with more implants. Regarding to previous studies immediate loading of implant in anterior mandible has predictable result and can use for eliminating the edentulous phase of patients.

\section{REFERENCES}

Attard, N.J. and G.A. Zarb, 2005. Immediate and early implant loading protocols: A literature review of clinical studies. J. Prosthet. Dent., 94: 242-258.

Bergendal, T. and B. Engquist, 1998. Implant-supported overdentures: A longitudinal prospective study. Int. J. Oral Maxillofac. Implants, 13: 253-262.

Cannizzaro, G., M. Leone, U. Consolo, V. Ferri and M. Esposito, 2008. Immediate functional loading of implants placed with flapless surgery versus conventional implants in partially edentulous patients: A 3-year randomized controlled clinical trial. Int. J. Oral Maxillofac. Implants, 23: 867-875.

Chiapasco, M. and C. Gatti, 2003. Implant-retained mandibular overdentures with immediate loading: A 3 - to 8-year prospective study on 328 implants. Clin. Implant Dent. Relat. Res., 5: 29-38.

Chiapasco, M., S. Abati, E. Romeo and G. Vogel, 2001. Implant-retained mandibular overdentures with branemark system MKII implants: A prospective comparative study between delayed and immediate loading. Int. J. Oral Maxillofac. Implants, 16: 537-546.

Cochran, D.L., 2006. The evidence for immediate loading of implants. J. Evid. Base Dent. Pract., 6: 155-163.

Ercoli, C., P.R. Romano, M. Al Mardini and L. Cordaro, 2006. Restoration of immediately placed implants in 3 appointments: From surgical placement to definitive prostheses. J. Prosthet. Dent., 96: 212-218.

Kronstrom, M. and B. Davis, 2010. Immediate functional loading of 1 or 2 dental implants supporting a ball retained mandibular overdenture: Interim results of a randomized prospective study. J. Oral Maxillofac. Surg., 68: e15-e15.
Lee, C.K. and J.R. Agar, 2006. Surgical and prosthetic planning for a two-implant-retained mandibular overdenture: A clinical report. J. Prosthet. Dent., 95: 102-105

Lee, J.Y., S.G. Kim, S.Y. Moon, S.C. Lim, J.L. Ong and K.M. Lee, 2009. A short-term study on immediate functional loading and immediate nonfunctional loading implant in dogs: Histomorphometric evaluation of bone reaction. Oral Surg. Oral Med. Oral Pathol. Oral Radiol. Endodontol., 107: 519-524.

Liddelow, G. and P. Henry, 2010. The immediately loaded single implant-retained mandibular overdenture: A 36-month prospective study. Int. J. Prosthodont., 23: $13-21$.

Liddelow, G.J. and P.J. Henry, 2007. A prospective study of immediately loaded single implant-retained mandibular overdentures: Preliminary one-year results. J. Prosthet. Dent., 97: S126-S137.

Misch, C. and M.W. Bidez, 2008. A Scientific Rational for Dental Implant Design. In: Implant Dentistry, Misch, C. (Ed.). 3nd Edn., Mosby, St. Louis.

Payne, A.G., A. Tawes-Smith, R. Kumara and W.M. Thomson, 2001. One-year prospective evaluation of the early loading of unsplinted conical branemark fixtures with mandibular overdentures immediately following surgery. Clin. Implant Dent. Relat. Res., 3: 9-19.

Sadowsky, S.J. and A.A. Caputo, 2004. Stress transfer of four mandibular implant overdenture cantilever designs. J. Prosthet. Dent., 92: 328-336.

Stephan, G., F. Vidot, R. Noharet and P. Mariani, 2007. Implant-retained mandibular overdentures: A comparative pilot study of immediate loading versus delayed loading after two years. J. Prosthet. Dent., 97: S138-S145.

Tokuhisa, M., Y. Matsushita and K. Koyano, 2003. In vitro study of a mandibular implant overdenture retained with ball, magnet, or bar attachments: Comparison of load transfer and denture stability. Int. J. Prosthet., 16: 128-134.

Winter, W., S.M. Heckmann and H.P. Weber, 2004. A time-dependent healing function for immediate loaded implants. J. Biomech., 37: 1861-1867. 